\title{
Prevalence of Positive QuantiFERON-TB Gold In-Tube Test in Uveitis and its Clinical Implications in a Country Nonendemic for Tuberculosis
}

\section{FAHRIYE GROEN-HAKAN, JAN A.M. VAN LAAR, MARLEEN BAKKER, P. MARTIN VAN HAGEN, HANNAH HARDJOSANTOSO, AND ANIKI ROTHOVA}

- PURPOSE: To report on the prevalence and clinical implications of positive QuantiFERON-Gold (QFT-G) test results in the diagnostic evaluation of a large cohort of consecutive patients with uveitis in the Netherlands.

- DESIGN: Retrospective cross-sectional study.

- METHODS: This study included 710 consecutive patients who all underwent evaluation for uveitis including QFT-G testing. The ocular features, comorbidity, and abnormalities in diagnostic imaging and laboratory tests were registered for QFT-G-positive patients with uveitis. - RESULTS: Of all patients, $13 \%(92 / 710)$ were positive for QFT-G. Previously treated tuberculosis (TB) was documented in 2 patients. Of all 92 QFT-G-positive patients, culture-proven active TB was observed in 1 case. The proportion of patients with uveitis of unknown etiology was higher in QFT-G-positive than in the QFT-G-negative patients $(54 / 92$, $59 \%$ vs $238 / 618$, $39 \% ; P=.0004)$. The uveitis features of QFT-G-positive patients were mainly nonspecific. Of all QFT-G-positive patients with uveitis, 17 patients had chest imaging changes suggesting either TB or sarcoidosis. Twentynine QFT-G-positive patients with otherwise unexplained uveitis completed antituberculous therapy (29/ $710 ; 4 \%$ of all included patients) with beneficial effect in most cases.

- CONCLUSION: The QFT-G tested positive in $13 \%$ of patients with uveitis in the Netherlands, whereas only sporadic patients had a documented previous or active TB infection. The proportion of patients with unexplained uveitis was higher in QFT-G-positive patients. Though the association between uveitis and a positive QFT-G test might be coincidental, the majority of treated QFT-G-positive patients with otherwise unexplained severe uveitis cause had a beneficial response to antituber-

Accepted for publication Nov 8, 2019

From the Department of Ophthalmology (F.G-H., H.H., A.R.), Department of Internal Medicine (J.A.M.v.L., P.M.v.H.), Section of Clinical Immunology, Department of Immunology (J.A.M.v.L., P.M.v.H.), Medical Immunology, and the Department of Pulmonology (M.B.), Erasmus Medical Center, Rotterdam, The Netherlands.

Inquiries to Fahriye Groen-Hakan, Department of Ophthalmology, Erasmus Medical Center, Erasmus University, Dr. Molewaterplein 50. 60, 3015 GE Rotterdam, The Netherlands; e-mail: f.groen@erasmusmc.nl culous therapy. (Am J Ophthalmol 2019; $\mathbf{\square}: \mathbf{\square}-\mathbf{\square}$. (C) 2019 Elsevier Inc. All rights reserved.)

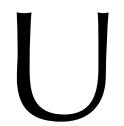

VEITIS MAY COMPLICATE AN ACTIVE TUBERCU. losis (TB) infection, but uveitis has often been reported to develop in patients with latent $\mathrm{TB}$ infections. The link between a latent TB infection and the development of uveitis was initially based on multiple case reports and a beneficial response of uveitis activity to antituberculous treatment (ATT) in a proportion of QuantiFERON-GOLD (QFT-G)-positive patients (Cellestis Limited, Carnegie, Victoria, Australia) with otherwise unexplained uveitis. ${ }^{1-8}$ Two recent prospective studies demonstrate an excess of idiopathic uveitis in the QFT-G-positive but otherwise healthy population, which favors a genuine link between uveitis and latent TB. ${ }^{9,10}$

The pathogenesis of uveitis in the setting of TB infection is not yet entirely understood. Active infection as well as immune-mediated reactions in the absence of replicating bacteria have both been suggested to play a role. ${ }^{1,9,10}$ Broncheoalveolar studies in a cohort of 109 patients with the positive tuberculin skin test and uveitis of undetermined cause have not demonstrated any active TB infection. ${ }^{11}$ In contrast, cultures positive for Mycobacterium tuberculosis were documented from lymph nodes detected on chest imaging in some QFT-G-positive patients with uveitis (but without any systemic complaints). ${ }^{12}$ Polymerase chain reaction (PCR) studies in intraocular fluid samples of patients with (presumed) TB-related uveitis was only occasionally positive for $\mathrm{M}$ tuberculosis. ${ }^{13}$

Patients with uveitis who are otherwise healthy and who test positive on an interferon gamma release assay cause a diagnostic and therapeutic dilemma. Uveitis in these patients might be related to their TB infection but could also be coincidental. The ocular characteristics previously related to latent TB-associated uveitis are serpiginouslike choroiditis, multifocal choroiditis, and retinal occlusive vasculitis. ${ }^{1,4,14-18}$

Most studies on uveitis in QFT-G-positive patients have included predominantly patients who have signs that are suggestive for TB-associated uveitis. The purpose of our study is to determine the prevalence of positive QFT-G 
testing in a large number of consecutive patients with uveitis in a nonendemic country and to report on the ocular and systemic features of patients with positive QFT-G tests.

\section{METHODS}

- PARTICIPANTS: Participants were adult patients with uveitis who underwent a diagnostic evaluation at the Erasmus University Medical Center in Rotterdam, The Netherlands, between January 2011 and July 2017. The medical ethical committee of Erasmus University Medical Center approved this retrospective cross-sectional study.

- ASSESSMENT OF CLINICAL CHARACTERISTICS: Anatomical classification of uveitis was performed according to the Standardization of Uveitis Nomenclature Working Group. ${ }^{19}$ The diagnostic evaluation of uveitis patients includes erythrocyte sedimentation rate, C-reactive protein, a complete blood cell count, serum angiotensinconverting enzyme (ACE; cut-off $68 \mathrm{U} / \mathrm{mL}$ ), and syphilis serology. In patients with anterior uveitis and panuveitis, human leukocyte antigen B27 was determined.

The QFT-G was introduced in the standard evaluation for uveitis in 2010 in our center (regardless of anatomic localization of uveitis and irrespective of a suspicion of TB-associated uveitis). QFT-G was considered positive according to the manufacturer's instructions $(\geq 0.35 \mathrm{IU} / \mathrm{mL})$.

The cause of uveitis was determined after the relevant diagnostic tests were performed and subsequently patients with uveitis were classified according to their QFT-G test result. Patients with uveitis of unknown cause despite the diagnostic evaluation but who had positive QFT-G test results were still classified as uveitis of unknown cause and separately indicated. Medical records of QFT-G-positive patients with uveitis were further investigated for systemic and ocular characteristics.

- ASSESSMENT OF CLINICAL CHARACTERISTICS IN QFTG-POSITIVE PATIENTS: A definitive diagnosis of TBassociated uveitis was made in patients with active systemic TB proven by a positive microbiology test anywhere in the body and without any other explanation of uveitis. ${ }^{13,14,19,20}$ After exclusion of other causes of uveitis, presumed active TB was diagnosed in 4 patients without positive culture and/or PCR sequencing, but with positive QFT-G test and clinical features typical of active TB disease (ie, constitutional symptoms, such as fevers, night sweats, and weight loss, as well as pulmonary and extrapulmonary signs suggesting active TB). ${ }^{20}$ Previous TB was defined as a history of documented treatment for TB. In our uveitis population, latent $\mathrm{TB}$ was considered in patients with positive QFT-G tests who were otherwise healthy and who did not exhibit signs of active TB.
A definitive diagnosis of sarcoidosis was considered in biopsy-confirmed cases in which other granulomatous diseases were excluded. Presumed sarcoidosis was considered in patients with chest imaging consistent with the diagnosis of sarcoidosis.

Serpiginous-like choroiditis was defined as patients with multiple serpiginous-like chorioretinal lesions not located adjacent to the optic disc. ${ }^{21-23}$

Results of ACE and soluble interleukin-2 receptor (sIL2R), if available, were also registered. Systemic comorbidities and outcomes of tissue biopsy specimens (whenever performed) were noted. ${ }^{24}$

Chest imaging was reviewed for all QFT-G-positive patients by 2 pulmonologists. This included 75 chest radiographs, 35 computed tomography scans, and 10 somatostatin receptor scintigraphy scans. Five of the computed tomography scans of the chest $(14 \%)$ were combined with fluor-18-deoxyglucose (FDG) positron emission tomography (PET) scanning.

Chest imaging findings consistent with the diagnosis of sarcoidosis were defined as symmetrical bilateral hilar and subcarinal lymph node enlargement and/or interstitial lung patterns (eg, micronodules with a typical perilymphatic distribution and upper lobe predominance). ${ }^{25,26}$ Findings suggestive of active TB were defined as parenchymal infiltrates (typically apical and posterior segments of the upper lobes or superior segments of the lower lobes) with or without cavitary lesions or unilateral hilar and/or mediastinal lymphadenopathy. Findings suggestive of healed TB were fibrotic scars, nodules with calcification, and pleural thickening (with or without calcification). ${ }^{19,25}$

All patients with (presumed) active TB were given ATT as well as all QFT-G-positive patients with severe, sight threatening uveitis in whom no other cause of uveitis could be established. ATT consisted of 3 drugs for patients with severe uveitis and a positive QFT-G test and no systemic localization of TB infection: isoniazid, rifampicin, and pyrazinamide for 2 months, followed by isoniazid and rifampicin for an additional 4 months. Patients with uveitis and evidence of active TB disease elsewhere also received ethambutol until isoniazid sensitivity was proven, according to national guidelines. In addition, QFT-G-positive patients (with established diagnoses other than TB) in anticipation of steroid-sparing agents also underwent ATT consisting of only rifampicin and isoniazid for 4 months to prevent reactivation of latent $\mathrm{TB}$ according to national guidelines. ${ }^{27}$

The threshold for the definition of a TB-endemic country was defined as an incidence of $>50$ cases per 100,000 population, according to the Dutch Guideline definition for TB risk groups. ${ }^{28,29}$

- STATISTICAL ANALYSIS: The mean, standard deviation, and range are reported for continuous measures. For categorical measures, percentages are reported, and the comparisons are performed using either the $\chi^{2}$ or Fisher exact 
TABLE 1. General Characteristics of Uveitis Patients

\begin{tabular}{|c|c|c|c|}
\hline & $\begin{array}{c}\text { Total } \\
(\mathrm{N}=710)\end{array}$ & $\begin{array}{l}\text { Positive QFT-G Test } \\
\qquad(\mathrm{n}=92)\end{array}$ & $\begin{array}{l}\text { Negative QFT-G Test } \\
\qquad(n=618)\end{array}$ \\
\hline \multicolumn{4}{|l|}{ Race, n (\%) } \\
\hline Nonwhite & 237 (33) & $58(63)$ & $179(29)$ \\
\hline White & $473(67)$ & $34(37)$ & $439(71)$ \\
\hline \multicolumn{4}{|l|}{ Gender, n (\%) } \\
\hline Male & $258(36)$ & $41(45)$ & $217(35)$ \\
\hline Female & $452(64)$ & $51(55)$ & $401(65)$ \\
\hline \multicolumn{4}{|l|}{ Anatomic localization, n (\%) } \\
\hline Anterior & $186(26)$ & $19(21)$ & $167(27)$ \\
\hline Intermediate & $62(9)$ & $3(3)$ & $59(10)$ \\
\hline Posterior & $193(27)$ & $25(27)$ & $168(27)$ \\
\hline Panuveitis & $231(33)$ & $40(43)$ & $191(31)$ \\
\hline Scleritis & $38(5)$ & $5(5)$ & $33(5)$ \\
\hline $\begin{array}{l}\text { Associated systemic disease } \\
\text { (noninfectious), n (\%) }\end{array}$ & $251(36)$ & $19(21)$ & $232(38)$ \\
\hline Sarcoidosis (biopsy proven) & $75(30)$ & $1(5)$ & $74(32)$ \\
\hline Sarcoidosis (presumed) & $37(15)$ & $6(32)$ & $31(13)$ \\
\hline Infection, $\mathrm{n}(\%)$ & $71(9)$ & $7(8)^{a}$ & $64(10)$ \\
\hline Clinical entity, $n(\%)$ & $96(14)$ & $12(13)$ & $84(14)$ \\
\hline Masquerade syndrome ${ }^{b}$ & $30(31)$ & $6(50)$ & $24(29)$ \\
\hline Unknown cause, n (\%) & $292(41)$ & $54(59)^{b}$ & $238(39)^{c}$ \\
\hline \multicolumn{4}{|c|}{$\begin{array}{l}\text { QTF-G = QuantiFERON-TB Gold; TB = tuberculosis. } \\
\text { ancluding presumed active tuberculosis }(n=4) \text {, active tuberculosis }(n=1) \text {, and toxoplasmosis }(n=2) \text {. } \\
\text { 'Including vitreoretinal lymphoma }(n=6) \text {, retinal vascular occlusions }(n=4) \text {, drusen }(n=2) \text {, degenerative vitreous floaters }(n=2) \text {, macular } \\
\text { dystrophy }(n=3) \text {, myopic degeneration }(n=2) \text {, retinitis pigmentosa }(n=2) \text {, Coats' disease }(n=1) \text {, ocular ischemic syndrome }(n=1) \text {, hyper- } \\
\text { tensive retinopathy }(n=1) \text {, chronic serous chorioretinopathy }(n=1) \text {, idiopathic intracranial hypertension }(n=1) \text {, retinal macroaneurysm }(n=1) \text {, } \\
\text { optic neuritis }(n=1) \text {, idiopathic macular telangiectasia }(n=1) \text {, and optic atrophy }(n=1) \text {. } \\
\text { 'The proportion of uveitis with unknown etiology was higher in QFT-G-positive than in QFT-G-negative patients (54/92 [59\%] vs 238/618 } \\
\text { [39\%]; } P=.0004) \text {. }\end{array}$} \\
\hline
\end{tabular}

tests. All $P$ values were 2 -tailed, and statistical significance was set at $P<.05$.

\section{RESULTS}

THE GENERAL CHARACTERISTICS OF OUR UVEITIS POPULAtion according to their QFT-G test results are shown in Table 1 . The population consisted mostly of white patients $(67 \%)$ with a slight female predominance $(64 \%)$. Ninetytwo patients of $710(13 \%)$ tested positive for QFT-G. QFT-G-positive patients were more often of nonwhite origin $(58 / 92 ; 63 \%)$ compared with QFT-G-negative patients $(179 / 618$ [29\%]; $P=.0001)$ but did not differ in gender $(P=.08)$.

The proportion of uveitis with unknown etiology was higher in QFT-G-positive than in QFT-G-negative patients (54/92 [59\%] vs 238/618 [39\%]; $P=.0004)$. Anatomic classification of uveitis was similar in QFT-Gpositive and QFT-G-negative patients, but panuveitis was more common in QFT-G-positive patients (40/92 [43\%] vs $191 / 618$ [31\%]; $P=.02$ ).

Overall, 38\% of QFT-G-positive patients were born outside the Netherlands in a TB-endemic country (Table 2). Age did not correlate with the QFT-G levels (Spearman $\rho$ correlation coefficient $-0.134, P=.203$ ). The median QFT-G value of QFT-G-positive patients was $3.45 \mathrm{U} / \mathrm{mL}$ (interquartile range [IQR] 9.8) and was different in QFT-G-positive patients with a known cause of uveitis when compared with the remainder of QFT-Gpositive patients with uveitis $(0.8 \mathrm{U} / \mathrm{mL}$ [IQR 8.5$]$ vs $6.8 \mathrm{U} / \mathrm{mL}$ [IQR 11.5]; $P=.06)$.

The characteristics of patients with QFT-G-positive uveitis are shown in Table 2. Documented previous TB was observed in 2 patients who received full ATT before developing uveitis (1 patient had human leukocyte antigen B27-associated uveitis and 1 patient had uveitis of unknown cause). Active TB was diagnosed in 1 patient and presumed active TB was diagnosed in 4 patients. The etiology of uveitis, other than (presumed) TB or sarcoidosis, was established in 26 of 92 (28\%) of QFTG-positive patients; no cause was identified in 54 
TABLE 2. Characteristics of Patients with Uveitis Positive in QuantiFERON-TB Gold Test

\begin{tabular}{|c|c|c|c|c|}
\hline & \multicolumn{4}{|c|}{ Positive QFT-G Test } \\
\hline & $\begin{array}{l}\text { Total } \\
(\mathrm{N}=92)\end{array}$ & $\begin{array}{l}\text { Uveitis of Unknown } \\
\text { Origin }(n=54)\end{array}$ & $\begin{array}{c}\text { (Presumed) TB or } \\
\text { Sarcoidosis }(n=12)^{a}\end{array}$ & $\begin{array}{l}\text { Uveitis with an Established Cause } \\
\text { Other than TB or Sarcoidosis }(n=26)^{b}\end{array}$ \\
\hline Mean age at uveitis onset, years $( \pm S D)$ & $46.1( \pm 15.5)$ & $45.8( \pm 15.2)$ & $48.2( \pm 17.3)$ & $45.9( \pm 15.2)$ \\
\hline \multicolumn{5}{|l|}{ Race, n (\%) } \\
\hline Nonwhite & $57(62)$ & $35(65)$ & $8(67)$ & $14(54)$ \\
\hline White & $35(38)$ & $19(35)$ & $4(33)$ & $12(46)$ \\
\hline \multicolumn{5}{|l|}{ Gender, n (\%) } \\
\hline Male & $41(45)$ & $21(39)$ & $3(25)$ & $17(65)$ \\
\hline Female & $51(55)$ & $33(61)$ & $9(75)$ & $9(35)$ \\
\hline \multicolumn{5}{|l|}{ Laterality, n (\%) } \\
\hline Unilateral & $37(40)$ & $22(41)$ & $4(33)$ & $11(42)$ \\
\hline Bilateral & $55(60)$ & $32(59)$ & $8(67)$ & $15(58)$ \\
\hline \multicolumn{5}{|l|}{ Anatomic localization, n (\%) } \\
\hline Anterior & $19(21)$ & $11(20)$ & $4(33)$ & $4(15)$ \\
\hline Intermediate & $3(3)$ & $2(4)$ & $1(8)$ & 0 \\
\hline Posterior & $25(27)$ & $13(24)$ & $1(8)$ & $11(42)$ \\
\hline Panuveitis & $40(43)$ & $23(43)$ & $6(50)$ & $11(42)$ \\
\hline Scleritis & $5(5)$ & $5(9)$ & 0 & 0 \\
\hline \multicolumn{5}{|l|}{ Specific ocular manifestations, n (\%) } \\
\hline Occlusive vasculitis & $7(8)$ & $6(11)$ & 0 & $1(4)^{c}$ \\
\hline Serpiginous-like choroiditis & $5(5)$ & $4(7)$ & $1(8)$ & 0 \\
\hline Serpiginous choroiditis & $2(2)$ & $2(4)$ & 0 & 0 \\
\hline Born outside the Netherlands, ${ }^{d} \mathrm{n}(\%)$ & $55(60)$ & $36(67)$ & $4(33)$ & $15(58)$ \\
\hline In TB-endemic country & $35(38)$ & $25(46)$ & $1(8)$ & $9(35)$ \\
\hline In country nonendemic for TB & $20(22)$ & $11(20)$ & $3(25)$ & $6(23)$ \\
\hline Born in the Netherlands, ${ }^{d} \mathrm{n}(\%)$ & $34(37)$ & $17(31)$ & $6(50)$ & $11(42)$ \\
\hline Stay $>6$ months in TB-endemic area & $4(12)$ & $4(24)$ & 0 & 0 \\
\hline QFT-G value $>5.0,{ }^{e} \mathrm{n}(\%)$ & $39(45)$ & $25(46)$ & $6(50)$ & $8(31)$ \\
\hline Median QFT-G value, U/mL (IQR) & $3.45(9.8)$ & $4.2(10.7)$ & $4.6(9.0)$ & $0.8(8.5)$ \\
\hline
\end{tabular}

$\mathrm{IQR}=$ interquartile range; $\mathrm{QFT}-\mathrm{G}=$ QuantiFERON-TB Gold; SD = standard deviation; TB = tuberculosis.

${ }^{a}$ Includes 1 patient with active TB. The patient with active TB-associated uveitis presented at 26 years of age with tuberculous psoas abscess (proven by cultures and polymerase chain reaction study) and uveitis for which he received full anti-TB treatment. Chest imaging during this period showed bilateral hilar lymphadenopathy. Four and a half years after the onset, he developed a recurrence of his uveitis and an abnormal mucosal lesion in his nose. A biopsy specimen was obtained, and it showed noncaseating granulomatous inflammation consistent with the diagnosis of sarcoidosis while polymerase chain reaction sequencing for Mycobacterium spp and cultures remained negative. Subsequent evaluation by a pulmonologist confirmed also pulmonary involvement with symmetrical hilar and mediastinal lymphadenopathy seen on a computed tomography scan of the chest. A biopsy specimen of the pulmonary lymph nodes showed granulomatous inflammation without any evidence of $M$ tuberculosis (negative culture and polymerase chain reaction sequencing). The soluble interleukin-2 receptor level was $8258 \mathrm{pg} / \mathrm{mL}$.

${ }^{b}$ This group consisted of Behçet disease $(n=4)$, human leukocyte antigen B27-associated uveitis $(n=4)$, Vogt-Koyanagi-Harada syndrome $(n=3)$, toxoplasmosis $(n=2)$, birdshot chorioretinopathy $(n=2)$, sympathetic ophthalmia $(n=1)$, lens-induced uveitis $(n=1)$, acute zonal occult outer retinopathy $(n=1)$, multiple sclerosis-associated uveitis $(n=1)$, and masquerade syndrome $(n=7)$.

${ }^{\mathrm{c}}$ This one patient had occlusive vasculitis because of Behçet disease.

dUnknown place of birth in 3/92 (3\%) of patients and unknown if the patient stayed in an TB endemic in 7/92 (8\%).

${ }^{e}$ Exact values were not available in 10 patients.

patients and the remaining 12 patients were diagnosed with (presumed) TB or sarcoidosis (Table 2). The ocular features of QFT-G-positive patients with uveitis of undetermined cause $(\mathrm{n}=54)$ were generally nonspecific. Serpiginous-like lesions were seen in 4 of 54 [7\%] and occlusive vasculitis in 6 of 54 [11\%], of which 3 were men between 30 and 45 years of age. Patients with serpiginous-like lesions and/or occlusive vasculitis had similar QFT-G levels compared with the remaining QFT-G-positive patients $(\geq 5.0 \mathrm{IU} / \mathrm{mL}$ in $6 / 13[46 \%]$ vs 33/79 [42\%]; $P=.77$ ).

ATT was initiated in 36 QFT-G-positive patients with severe uveitis of unknown origin. Of these, 7 did not complete ATT because of side effects resulting in 29 of 36 
TABLE 3. Chest Imaging and Laboratory Results in Patients with Uveitis Positive in QuantiFERON-TB Gold Test

\begin{tabular}{|c|c|c|c|c|}
\hline & \multicolumn{4}{|c|}{ Positive QFT-G Test } \\
\hline & $\begin{array}{l}\text { Total } \\
(\mathrm{N}=92)\end{array}$ & $\begin{array}{l}\text { Uveitis of Unknown } \\
\text { Origin }(n=54)\end{array}$ & $\begin{array}{l}\text { (Presumed) TB or } \\
\text { Sarcoidosis } \\
(n=12)\end{array}$ & $\begin{array}{l}\text { Uveitis with an Established } \\
\text { Cause Other than TB or } \\
\text { Sarcoidosis }(n=26)\end{array}$ \\
\hline Total imaging available, $\mathrm{n}(\%)$ & $78(85)$ & $50(93) \mathrm{v}$ & $12(100)$ & $16(62)$ \\
\hline $\begin{array}{l}\text { Total chest imaging consistent with } \\
\text { sarcoidosis and/or } \text { TB }^{a}\end{array}$ & $17(22)$ & $5(10)$ & $12(100)$ & 0 \\
\hline Tissue biopsy available, $\mathrm{n}(\%)$ & $9(10)^{b}$ & $4(7)$ & $4(33)$ & $1(4)$ \\
\hline Consistent with TB & 0 & 0 & 0 & 0 \\
\hline Consistent with sarcoidosis & $1(100)$ & 0 & $1(33)^{C}$ & 0 \\
\hline ACE available, $n(\%)$ & $73(79)$ & $43(80)$ & $12(100)$ & $18(69)$ \\
\hline ACE $\geq 68 \mathrm{U} / \mathrm{L}$ & $14(19)$ & $7(16)$ & $6(50)$ & $1(6)$ \\
\hline sIL-2R available, $n(\%)$ & $43(47)$ & $29(54)$ & $9(75)$ & $5(19)$ \\
\hline sIL-2R $\geq 4000 \mathrm{pg} / \mathrm{mL}$ & $21(49)$ & $10(34)$ & $9(100)$ & $2(40)$ \\
\hline \multicolumn{5}{|c|}{$\begin{array}{l}\text { ACE }=\text { angiotensin-converting enzyme; QFT-G = QuantiFERON-TB Gold; sIL-2R = soluble interleukin-2 receptor; TB = tuberculosis. } \\
{ }^{a} \text { Of these, } 11 \text { were detected by positron emission tomography/computed tomography scan, } 1 \text { by somatostatin receptor scintigraphy scan, } 3 \\
\text { with both radiography and positron emission tomography/computed tomography scan of the chest, and } 2 \text { by both positron emission tomog- } \\
\text { raphy/computed tomography scan of the chest and somatostatin receptor scintigraphy scan. } \\
{ }^{b} \text { Including biopsy of hilar lymph nodes }(n=5) \text {, histologic examination of enucleated eye }(n=2) \text {, lymph node located in the neck ( } n=1 \text { ), and } \\
\text { skin biopsy }(n=1) \text {. } \\
{ }^{c} \text { This patient was diagnosed with sarcoidosis by biopsy of hilar lymph nodes which showed granulomatous inflammation, negative polymer- } \\
\text { ase chain reaction sequencing for Mycobacterium tuberculosis spp, negative tuberculosis culture, and negative auramine stains. The biopsy } \\
\text { specimen obtained from the patient with active TB who was later diagnosed with sarcoidosis was not taken into account in this table, as his- } \\
\text { tologic evidence of sarcoidosis was gained after treatment for TB. }\end{array}$} \\
\hline
\end{tabular}

patients who completed ATT ( $81 \%$ of all with intention to treat; 29/54 [54\%] of all QFT-G-positive patients with uveitis of unknown etiology).

Of 29 fully treated patients, 16 (55\%) were without uveitis activity at the time of ATT completion and $20(69 \%)$ were quiet at 1 year of follow-up. However, 12 (41\%) needed systemic immunomodulatory drugs and/or periocular corticosteroid injections during ATT because of their ongoing uveitis activity. Of nontreated QFT-G-positive patients with uveitis of unknown origin, $13(100 \%)$ patients (followed for $\geq 1$ year after QFT-G testing) were without inflammatory activity at 1 year of follow-up and 7 (58\%) patients (followed for $\geq 18$ months after QFT-G testing) were without inflammatory activity at 18 months of follow-up. Nevertheless, 5 (42\%) patients needed systemic immunomodulatory drugs and/or periocular corticosteroid injections in this period because of their ongoing uveitis activity.

Abnormal radiologic features suggesting the diagnosis of sarcoidosis and/or TB were observed in 17 of 66 (26\%) of QFT-G-positive patients with an undetermined cause of uveitis (Table 3). Four patients had a biopsy specimen obtained from their enlarged hilar lymph nodes, but none of the tests performed was positive for $M$ tuberculosis (negative PCR for M tuberculosis spp and negative cultures and/or stains). FDG accumulation was observed in the mediastinal or hilar lymph nodes in all 5 performed
FDG-PET/computed tomography scans. Of these, 3 fulfilled the criteria for the diagnosis of sarcoidosis and 1 had intraocular lymphoma. The remaining patient was diagnosed with presumed active TB and showed nonspecific intrathoracic and intraabdominal generalized lymphadenopathy. The QFT-G level in this patient was 15.18 $\mathrm{IU} / \mathrm{mL}$ and the sIL-2R level was elevated $(6200 \mathrm{pg} / \mathrm{mL})$. A biopsy specimen of the pulmonary lymph nodes was obtained, and the specimen revealed an absence of granulomatous inflammation and negative results for $M$ tuberculosis.

Serum ACE was elevated in 14 of 73 (19\%) patients and sIL-2R was elevated in 21 of 43 [49\%] of QFT-G-positive patients. Serum ACE and QFT-G test levels were associated with each other (Spearman $\rho$ correlation coefficient 0.294; $P=.004,2$-sided); sIL-2R and QFT-G test levels were not (Spearman $\rho$ correlation coefficient $0.091 ; P=.39$ ).

\section{DISCUSSION}

THIS RETROSPECTIVE STUDY REVEALED POSITIVE QFT-G test results in $13 \%$ of a large Dutch uveitis cohort, of which only few had (presumed) active TB. An excess 
of unexplained uveitis was observed in QFT-G-positive patients, suggesting an association between latent $\mathrm{TB}$ infection and uveitis at least in a part of QFT-G-positive patients.

Only 1 patient in the QFT-G-positive group had concurrent culture-proven TB, which is similar to other studies from non-TB-endemic countries. ${ }^{30}$ In 54 of 92 (59\%) of QFT-G-positive patients, no cause for uveitis could be identified and therefore their uveitis could be related to a previous TB infection.

A positive QFT-G test indicates a true infection with $M$ tuberculosis but cannot distinguish between active and latent TB infections. The laboratory discrimination between these 2 conditions is currently not feasible. Therefore, the mere presence of a positive QFT-G test is not enough evidence for causation. Especially in endemic countries, the prevalence of positive QFT-G is high and the association with uveitis can be coincidental. Also in countries that are nonendemic for $\mathrm{TB}$, a high prevalence $(\leq 23 \%)$ of QFT-G positivity in the uveitis population was observed; howevern these studies included predominantly patients with clinical suspicion of ocular TB. ${ }^{12,31-34}$ Nonetheless, a disproportionally high prevalence of QFT-G-positive uveitis patients was observed in the present series, even higher than reported in Dutch high-risk groups $(0.01 \%$ in high-risk groups, eg, TB contacts). ${ }^{35,36}$

The pathogenesis of uveitis in the setting of latent TB infection is not yet entirely understood. Active TB infection affecting the eye was repeatedly suggested, which is supported by previous studies that demonstrated positive $\mathrm{M}$ tuberculosis cultures in lymph nodes of QFT-G-positive patients with uveitis without any other symptoms, suggesting active extraocular TB. However, cultures and/or PCR on ocular fluid samples remain negative in most cases and Mycobacteria are typically difficult to find by histologic examination, reflecting that ocular TB represents a paucibacillary inflammation. ${ }^{37}$ Therefore, the diagnosis of presumed ocular TB was proposed in the presence of ocular findings suggestive of tuberculosis (ie, choroidal granuloma, broad-based posterior synechiae, retinal vasculitis with or without choroiditis, or serpiginous-like choroiditis) combined with evidence of replicating bacilli elsewhere in the body. ${ }^{38}$ However, most of our QFT-G-positive patients did not fulfill these clinical ocular criteria, which might be explained by inclusion bias of previous studies. Our study encompassed all consecutive patients with uveitis, which might explain a relative lack of ocular signs typical for presumed TB. This phenomenon was also noted in prospective studies from endemic countries. ${ }^{9,10}$ An alternative theory about the pathogenesis in TB-associated uveitis is an immune reaction to $\mathrm{TB}$ antigens and subsequent cross-reaction with ocular antigens causing autoimmunity, though the evidence of such process is lacking. ${ }^{39}$

Chest imaging suggesting the diagnosis of $\mathrm{TB}$ and/or sarcoidosis in was noted in 17 (18\%) of QFT-G-positive patients, but our results do not provide information about the infectious or noninfectious causes of these abnormalities. The presence of pulmonary changes suggesting sarcoidosis might be explained by the fact that sarcoidosis can be triggered by persistent presentation of poorly degradable antigens, including $M$ tuberculosis, and the subsequent host response to it. ${ }^{40-43}$

A positive QFT-G test in a patient with uveitis does not confirm that uveitis is of TB origin. Hence, a response to ATT might aid in the diagnosis of TB-associated uveitis. ${ }^{38}$ However, ATT represents a lengthy treatment and might be associated with adverse effects. In our institution, all patients with active extraocular $\mathrm{TB}$ receive ATT and we recommend ATT to all QFT-G-positive patients with severe and sight-threatening uveitis. In the remaining cases with uveitis not responding to local treatment, a shared decision with the patient regarding ATT is taken. Herein, the ophthalmologist explains the possible (but not certain) relationship of uveitis and TB, informs the patient about the potential cure of uveitis with ATT, and discusses the possible adverse effects. The patient decides whether he or she will undergo the treatment. In consequence, the exact efficacy of ATT treatment in our study cannot be reliably evaluated: only patients with severe uveitis received ATT, and the numbers of QFT-G-positive patients treated with ATT were limited. In consequence, QFT-G-positive patients who did not receive ATT had milder uveitis, which might explain their beneficial outcomes. Our study is limited by the retrospective collection of data and the variable length of follow-up. As in all retrospective studies, the heterogeneity of investigations was documented and there were no systematic indications for performing computed tomography scans and biopsy procedures. The strength of our study lies in the large cohort of consecutive patients with uveitis tested by QFT-G, without a selection of patients with clinical suspicion of ocular TB. The results of QFT-G testing are also relevant for further management of patients with uveitis who require systemic immunosuppressive treatment regimens, such as biologic drugs.

In conclusion, the major findings of this study include the high prevalence of $13 \%$ of positive QFT-G tests in a Dutch uveitis population and an excess of unexplained uveitis in QFT-G-positive patients. We conclude that QFT-G testing is useful in the evaluation for uveitis in nonendemic countries because the QFT-G-positive patients with uveitis of otherwise unexplained origin might benefit from ATT, especially those with severe and sightthreatening uveitis. 
ALL AUTHORS HAVE COMPLETED AND SUBMITTED THE ICMJE FORM FOR DISCLOSURE OF POTENTIAL CONFLICTS OF INTEREST. Funding/Support: Dr Groen-Hakan was supported in part by a grant from AbbVie, the Netherlands to the Department of Ophthalmology, Erasmus Medical Center, Erasmus University, Rotterdam, the Netherlands. This sponsor had no role in the study design, the collection, analysis, and interpretation of data, nor in the writing of this report and in the decision to submit the article for publication. Financial Disclosures: The authors indicate no financial conflicts of interest. This study was designed, directed, and coordinated by A.R. and F.G-H. F.G-H., H.H., M.B., J.v.L., and A.R. reviewed patient charts' and analyzed data. The manuscript was written by F.G-H. and A.R. and commented on by all authors. All authors attest that they meet the current ICMJE criteria for authorship. We thank A. Koopmans (Erasmus Medical Center, Rotterdam, The Netherlands) for critically reviewing the manuscript and W. Drabarek (Erasmus Medical Center, Rotterdam, The Netherlands) for statistical assistance.

\section{REFERENCES}

1. La Distia Nora R, van Velthoven ME, Ten Dam-van Loon $\mathrm{NH}$, et al. Clinical manifestations of patients with intraocular inflammation and positive QuantiFERON-TB gold in-tube test in a country nonendemic for tuberculosis. Am J Ophthalmol 2014;157(4):754-761.

2. Bansal R, Gupta A, Gupta V, Dogra MR, Bambery P, Arora SK. Role of anti-tubercular therapy in uveitis with latent/manifest tuberculosis. Am J Ophthalmol 2008;146(5): $772-779$.

3. Al-Mezaine HS, Al-Muammar A, Kangave D, Abu ElAsrar AM. Clinical and optical coherence tomographic findings and outcome of treatment in patients with presumed tuberculous uveitis. Int Ophthalmol 2008;28(6):413-423.

4. Gupta V, Gupta A, Arora S, Bambery P, Dogra MR, Agarwal A. Presumed tubercular serpiginouslike choroiditis - Clinical presentations and management. Ophthalmology 2003;110(9):1744-1749.

5. Rosen PH, Spalton DJ, Graham EM. Intraocular tuberculosis. Eye (Lond) 1990;4(part 3):486-492.

6. Gupta A, Gupta V, Arora S, Dogra MR, Bambery P. PCRpositive tubercular retinal vasculitis: clinical characteristics and management. Retina 2001;21(5):435-444.

7. Morimura Y, Okada AA, Kawahara S, et al. Tuberculin skin testing in uveitis patients and treatment of presumed intraocular tuberculosis in Japan. Ophthalmology 2002;109(5): $851-857$.

8. van Daele PL, Bakker M, van Hagen PM, Baarsma GS, Kuijpers RW. TB or not TB: treat to see. Med J Aust 2006; 185(3):178-179.

9. La Distia Nora R, Sitompul R, Bakker M, et al. Tuberculosis and other causes of uveitis in Indonesia. Eye (Lond) 2018; 32(3):546-554.

10. Pathanapitoon K, Kunavisarut P, Sirirungsi W, Rothova A. Looking for ocular tuberculosis: prevalence and clinical manifestations of patients with uveitis and positive QuantiFERON((R))-TB Gold test. Ocul Immunol Inflamm 2018;26(6):819-826.

11. Caspers L, Makhoul D, Ebraert H, Michel O, Willermain F. Clinical manifestations of patients with intraocular inflammation and positive QuantiFERON-TB gold in-tube test in a country nonendemic for tuberculosis. Am J Ophthalmol 2014;158(3):646-647.

12. Doycheva D, Deuter C, Hetzel J, et al. The use of positron emission tomography/CT in the diagnosis of tuberculosis-associated uveitis. Br J Ophthalmol 2011; 95(9):1290-1294.

13. Agarwal A, Agrawal R, Gunasekaran DV, et al. The Collaborative Ocular Tuberculosis Study (COTS)-1 report 3: polymerase chain reaction in the diagnosis and management of tubercular uveitis: global trends. Ocul Immunol Inflamm 2019;27(3):465-473.

14. Gupta A, Bansal R, Gupta V, Sharma A, Bambery P. Ocular signs predictive of tubercular uveitis. Am J Ophthalmol 2010; 149(4):562-570.

15. De Luigi G, Mantovani A, Papadia M, Herbort CP. Tuberculosis-related choriocapillaritis (multifocal-serpiginous choroiditis): follow-up and precise monitoring of therapy by indocyanine green angiography. Int Ophthalmol 2012;32(1): 55-60.

16. Zhang M, Zhang J, Liu Y. Clinical presentations and therapeutic effect of presumed choroidal tuberculosis. Retina 2012;32(4):805-813.

17. Davis EJ, Rathinam SR, Okada AA, et al. Clinical spectrum of tuberculous optic neuropathy. J Ophthalmic Inflamm Infect 2012;2(4):183-189.

18. Sudharshan S, Ganesh SK, Balu G, et al. Utility of QuantiFERON(R)-TB Gold test in diagnosis and management of suspected tubercular uveitis in India. Int Ophthalmol 2012;32(3):217-223.

19. Jabs DA, Nussenblatt RB, Rosenbaum JT, Nomenclatu SU. Standardization of uveitis nomenclature for reporting clinical data. Results of the First International Workshop. Am J Ophthalmol 2005;140(3):509-516.

20. American Thoracic Society. Diagnostic standards and classification of tuberculosis. Am Rev Respir Dis 1990;142(3): 725-735.

21. Nazari Khanamiri H, Rao NA. Serpiginous choroiditis and infectious multifocal serpiginoid choroiditis. Surv Ophthalmol 2013;58(3):203-232.

22. Bansal R, Gupta A, Gupta V, Dogra MR, Sharma A, Bambery P. Tubercular serpiginous-like choroiditis presenting as multifocal serpiginoid choroiditis. Ophthalmology 2012;119(11):2334-2342.

23. Vasconcelos-Santos DV, Rao PK, Davies JB, Sohn EH, Rao NA. Clinical features of tuberculous serpiginouslike choroiditis in contrast to classic serpiginous choroiditis. Arch Ophthalmol 2010;128(7):853-858.

24. Groen-Hakan F, Eurelings L, ten Berge JC, et al. Diagnostic value of serum-soluble interleukin 2 receptor levels vs angiotensin-converting enzyme in patients with sarcoidosisassociated uveitis. JAMA Ophthalmol 2017;135(12): 1352-1358.

25. Spagnolo P, Rossi G, Trisolini R, Sverzellati N, Baughman RP, Wells AU. Pulmonary sarcoidosis. Lancet Respir Med 2018;6(5):389-402.

26. Naidich D. Are CT findings of pulmonary sarcoidosis ever sufficient for a presumptive diagnosis? Lancet Respir Med 2018;6(9):e43. 
27. Bakker M, Boeree MJ, de Lange WCM, van LoenhoutRooyackers JH, de Vries G. Richtlijn Medicamenteuze behandeling van tuberculose 2014. Available at https://www.nvalt.nl/ kwaliteit/richtlijnen/infectieziekten//Infectieziekten/Richtlijnmedicamenteuze-behandeling-van-TBC\%200ktober\%202014. pdf. Accessed April 12, 2019.

28. World Health Organization. Country profiles for 30 high TB burden countries. Available at http://www.who.int/ tb/publications/global_report/gtbr2017_annex2.pdf?ua=1. Accessed April 12, 2019.

29. World Health Organization. Use of high burden country lists for TB by WHO in the post-2015 era. Available at https:// www.who.int/tb/publications/global_report/high_tb_burden countrylists2016-2020.pdf. Accessed April 12, 2019.

30. Trad S, Bodaghi B, Saadoun D. Update on immunological test (Quantiferon-TB Gold) contribution in the management of tuberculosis-related ocular inflammation. Ocul Immunol Inflamm 2018;26(8):1192-1199.

31. Jakob E, Max R, Zimmermann S, et al. Three years of experience with QuantiFERON-TB gold testing in patients with uveitis. Ocul Immunol Inflamm 2014;22(6):478-484.

32. Mackensen F, Becker MD, Wiehler U, Max R, Dalpke A, Zimmermann S. QuantiFERON TB-Gold — a new test strengthening long-suspected tuberculous involvement in serpiginouslike choroiditis. Am J Ophthalmol 2008;146(5):761-766.

33. Gineys R, Bodaghi B, Carcelain G, et al. QuantiFERON-TB gold cut-off value: implications for the management of tuberculosis-related ocular inflammation. Am J Ophthalmol 2011;152(3):433-440.e431.

34. Cordero-Coma M, Calleja S, Torres HE, et al. The value of an immune response to Mycobacterium tuberculosis in patients with chronic posterior uveitis revisited: utility of the new IGRAs. Eye 2010;24(1):36-43.

35. Erkens CG, Slump E, Verhagen M, et al. Monitoring latent tuberculosis infection diagnosis and management in the Netherlands. Eur Respir J 2016;47(5):1492-1501.

36. Tuberculose in Nederland 2016. Availale at https://www.rivm. nl/sites/default/files/2018-11/TiN\%202016\%20surveillancer apport.pdf. Accessed December 20, 2018.

37. Wroblewski KJ, Hidayat AA, Neafie RC, Rao NA, Zapor M. Ocular tuberculosis: a clinicopathologic and molecular study. Ophthalmology 2011;118(4):772-777.

38. Gupta A, Sharma A, Bansal R, Sharma K. Classification of intraocular tuberculosis. Ocul Immunol Inflamm 2015;23(1):7-13.

39. La Distia Nora R, Ten Berge JC, Rothova A, Schreurs MW. Antinuclear and antiretinal antibodies in uveitis associated with active and latent tuberculosis. Acta Ophthalmol 2018; 96(5):e659-e660.

40. Perez RL, Rivera-Marrero CA, Roman J. Pulmonary granulomatous inflammation: from sarcoidosis to tuberculosis. Semin Respir Infect 2003;18(1):23-32.

41. Papaetis GS, Pefanis A, Solomon S, Tsangarakis I, Orphanidou D, Achimastos A. Asymptomatic stage I sarcoidosis complicated by pulmonary tuberculosis: a case report. J Med Case Rep 2008;2:226.

42. Mise K, Goic-Barisic I, Puizina-Ivic N, Barisic I, Tonkic M, Peric I. A rare case of pulmonary tuberculosis with simultaneous pulmonary and skin sarcoidosis: a case report. Cases J 2010;3:24.

43. Sarkar S, Saha K, Das CS. Isolated tuberculous liver abscess in a patient with asymptomatic stage I sarcoidosis. Respir Care 2010;55(12):1751-1753. 